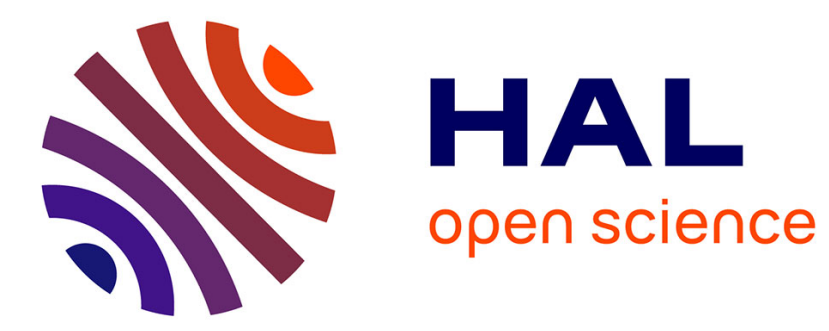

\title{
Photofragmentation of cyanogen upon carbon and nitrogen K-shell excitation by partial ion yield experiments
}

G Ohrwall, W C Stolte, R Guillemin, S.-W Yu, M N Piancastelli, D W Lindle

\section{- To cite this version:}

G Ohrwall, W C Stolte, R Guillemin, S.-W Yu, M N Piancastelli, et al.. Photofragmentation of cyanogen upon carbon and nitrogen $\mathrm{K}$-shell excitation by partial ion yield experiments. Journal of Physics B: Atomic, Molecular and Optical Physics, 2010, 43 (9), pp.95201. 10.1088/09534075/43/9/095201 . hal-00569915

\section{HAL Id: hal-00569915 https://hal.science/hal-00569915}

Submitted on 25 Feb 2011

HAL is a multi-disciplinary open access archive for the deposit and dissemination of scientific research documents, whether they are published or not. The documents may come from teaching and research institutions in France or abroad, or from public or private research centers.
L'archive ouverte pluridisciplinaire HAL, est destinée au dépôt et à la diffusion de documents scientifiques de niveau recherche, publiés ou non, émanant des établissements d'enseignement et de recherche français ou étrangers, des laboratoires publics ou privés. 


\title{
Photofragmentation of cyanogen upon carbon and nitrogen K-shell excitation by partial ion yield experiments.
}

\author{
G. Öhrwall ${ }^{1}$, W. C. Stolte ${ }^{2,3}$, R. Guillemin ${ }^{4,5}$, S.-W. Yu ${ }^{6}, \mathbf{M}$. \\ N. Piancastelli ${ }^{7}, \mathrm{D}$. W. Lindle ${ }^{2}$ \\ 1 MAX-lab, Lund University, Box 118, SE-221 00 Lund, Sweden \\ ${ }^{2}$ Department of Chemistry, University of Nevada, Las Vegas, NV 89154-4003, USA \\ 3 Advanced Light Source, Lawrence Berkeley National Laboratory, Berkeley, \\ California 94720 \\ ${ }^{4}$ CNRS, UMR 7614, Laboratoire de Chimie Physique Matière et Rayonnement, \\ F-75005 Paris, France \\ ${ }^{5}$ UPMC Univ Paris 06, UMR 7614, Laboratoire de Chimie Physique Matière et \\ Rayonnement, F-75005 Paris, France \\ ${ }^{6}$ Lawrence Livermore National Laboratory, Livermore, CA 94550, USA \\ 7 Department of Physics and Materials Science, Uppsala University, Box 530, SE-751 \\ 21 Uppsala, Sweden
}

\begin{abstract}
Partial ion yield spectroscopy was used to study fragmentation processes in cyanogen after $\mathrm{C} 1 s$ and $\mathrm{N} 1 s$ photoexcitation. The interpretation of the core excitation spectrum mostly follows literature, but the high resolution has in some cases allowed a more detailed assignment. Using the fact that in Rydberg states there is an intensity increase as the fragmentation process becomes more extensive compared to valence orbitals, we have been able to distinguish resonances with valence and Rydberg character. Furthermore, we have been able to assign features in the ionization continuum as arising from double excitations or shape resonances, by observation of the suppression of anion yield at shape resonances.
\end{abstract}




\section{Introduction}

Cyanogen, $(\mathrm{CN})_{2}$, is a stable gas while its subunit, the radical $\mathrm{CN}$, is of high interest in astrophysical studies, being found in comet tails and nuclei. The amount and distribution of $\mathrm{CN}$ in the Hale Bopp comet revealed some crucial details about the mechanisms controlling the evolution of comets [1], with the CN fluorescence spectrum being used to determine comet velocity and rotational properties [2]. To elucidate the electronic structure of its stable parent $(\mathrm{CN})_{2}$ is therefore quite relevant from both a fundamental and an applied point of view. Several studies have been reported on $(\mathrm{CN})_{2}$ electronic structure, including He I and He II valence photoelectron spectra $[3,4,5]$, a synchrotron radiation based pulsed field ionization study [6], and theoretical calculations of the valence electronic structure with several methods $[7,8,9,10]$. However, no extensive investigation is reported on the core excitation and decay properties, the only existing literature study being an electron energy loss spectroscopy study of the absorption around both the $\mathrm{C}$ and the $\mathrm{N} K$-edges [11].

We present here an investigation of the electronic structure and dynamical properties of molecular cyanogen by positive and negative ion partial yield spectroscopy, with the main goal of elucidating its resonant behavior and fragmentation properties around both $\mathrm{C}$ and $\mathrm{N} K$-edges. We have conducted a systematic study of partial positive and negative ion yields on as many as twenty molecules, which has allowed us to establish several criteria for spectral assignment based upon some peculiar features of partial ion yield experiments. Among these are $\mathrm{CO}[12,13], \mathrm{CO}_{2}$ [14], $\mathrm{H}_{2} \mathrm{O}[15]$, OCS [16], $\mathrm{N}_{2} \mathrm{O}$ [17], $\mathrm{NO}$ [18], $\mathrm{H}_{2} \mathrm{~S}$ [19], and $\mathrm{CHOOH}$ [20]. In particular, we have demonstrated that in relatively small systems the anion yield in correspondence of shape resonance-related spectral features is negligible, due to the difficulty of producing a negatively charged species starting from a species with a double positive charge, resulting from mechanisms like normal Auger decay [12]. Such a finding has proven a very valuable tool in cases where the presence of shape resonances was controversial, or in cases where there was an overlap in energy between shape resonances and doubly excited states embedded in an ionization continuum. Shape resonances are well known phenomena in molecular photoionization, and have been studied extensively in the last decades, in particular after the emergence of synchrotron radiation facilities. The first described case was the $l=3$ shape resonance in the $(1 s)^{-1} \sigma_{u}$ continuum of $\mathrm{N}_{2}$, discussed in a Letter by Dehmer and Dill published nearly 30 years ago [21]. There have been instances where the assignment of certain features in photoionization cross-section spectra to shaperesonant phenomena or not have been debated. This highlights the need to be able to unambiguously assign features above the threshold in core photoionization spectra as arising from shape resonances, or double excitations, i.e., two-hole-two-electron coreexcited states, or correlation satellite thresholds. The key question in assigning a shape resonance is ascertaining the one-electron character of the feature, which for instance can be made by measuring the cross section for only the main line. Recently, we showed that 
for a small molecule such as $\mathrm{CO}$, an alternative method is to measure the production of anions after core excitation [12, 13]. For light elements such as carbon or oxygen, the decay following the excitation of the core electron into the ionization continuum will almost always include the ejection of an Auger electron. This leaves the CO ion in a doubly charged state, from which ion-pair formation is not possible. From doubly excited states above the threshold, the resonant Auger process allows decay to singly charged final states, which may form ion pairs upon dissociation in a subsequent step.

The situation becomes more difficult to interpret for larger molecules. In the twoand three-atomic cases mentioned above [12, 13, 14, 15, 16, 17, 18, 19], anion yield has been found to be suppressed at shape resonances. However, in the case of SF6, anion yield of similar relative intensity as in cations has been observed at the position of the shape resonance, both by Scully et al. [22], and by some of us [23]. The possibility to distribute charge over several positive fragments opens up decay paths that will allow anions to be formed even after normal Auger decay. The present investigation thus also provides information on if the four-atomic molecule $(\mathrm{CN})_{2}$ is small enough to allow this criterion to apply.

In this framework, one of the reasons to select $(\mathrm{CN})_{2}$ is the existing rich literature on the presence, number and energy position of shape resonances. Cyanogen is a linear molecule with two strong triple bonds, for which a $l=3$ shape resonance, similar to $\mathrm{N}_{2}$, can be expected. However, as there are two pairs of core levels, both an ungerade and a gerade combination should be possible to form. Theoretical considerations for the ionization of valence orbitals have therefore shown a very complex picture concerning the existence of shape resonances in $(\mathrm{CN})_{2}[24,25,26]$. Another reason to perform a study on $(\mathrm{CN})_{2}$ is that the only reported observation of transitions to either Rydberg states or virtual molecular orbitals was obtained by moderate-resolution electron energy loss spectroscopy around both the $\mathrm{C}$ and the $\mathrm{N}$ K-edges [11]. In this literature report, the experimental resolution was not sufficient to resolve the Rydberg states overlapping with empty molecular orbitals. Another open question was whether there is a mixing of valence virtual states of sigma symmetry with the $3 s$ Rydberg level, as has been reported for instance on acetylene, another molecule with a strong triple bond [27]. A useful criterion we have been able to establish is that the relative intensity of spectral features in partial ion yield measurements related to states with a pure Rydberg character increases with respect to features related to transitions to virtual molecular orbitals as a function of fragmentation: the more extended the fragmentation process is, the more prominent the Rydberg series. This finding was explained on the ground of the transitions to Rydberg states leading mainly to spectator decay, therefore to final states often dissociative in nature (see e.g. [27, 28]). By using the method described above, we could identify several above-threshold features in $(\mathrm{CN})_{2}$ as either shape resonances or doubly excited states. In particular, we show that the enhancement in intensity at threshold for both edges is most likely due to doubly excited states converging to the ionization threshold of satellites rather than a shape resonance, on the ground of the 
behavior of the negative ion yields. Furthermore, we could perform a new assignment of the Rydberg series based on our increased spectral resolution together with the above described effect on the relative intensity of the Rydberg series as a function of extended fragmentation. A more complete picture of the dynamical properties of $(\mathrm{CN})_{2}$ is therefore presented here, derived mainly by exploiting some specific features of partial ion yield spectroscopy. We also report and discuss the surprising formation of

$\mathrm{N}_{2}^{+}$fragments. The formation of such fragment requires a strong rearrangement of the molecular structure following core excitation.

\section{Experimental}

The measurements were performed using undulator beamline 8.0.1.3 at the Advanced Light Source (Lawrence Berkeley National Laboratory, Berkeley, CA). This 5-cm-period undulator beamline is equipped with three interchangeable spherical gratings $(150,380$, and 925 lines $/ \mathrm{mm}$ ) for high resolution and high flux and provides $10^{11}$ to $6 \times 10^{15}$ photons/s in the $80-1400 \mathrm{eV}$ photon-energy range with a maximum resolving power of $E / \Delta E<8000$. Calibration of the photon energy was performed by measuring the position of the $\mathrm{C} 1 s \rightarrow 2 \pi_{u}$ in $\mathrm{CO}_{2}(290.77 \mathrm{eV}$ [29]) at the $\mathrm{C} K$-edge, and the position of the $\mathrm{N} 1 s \rightarrow 2 \pi_{g}$ resonance in $\mathrm{N}_{2}(400.87 \mathrm{eV}$ [30]) at the $\mathrm{N} K$-edge. Grating and slit sizes were chosen to achieve a resolution of $70 \mathrm{meV}$ at $300 \mathrm{eV}$ and $80 \mathrm{meV}$ at $400 \mathrm{eV}$, while preserving the photon flux (approximately $5 \times 10^{12}$ photons $/ \mathrm{s}$ ) to allow for a reasonable anion signal. Contamination by third-order light from the undulator passing through the monochromator accounts for less than $1 \%$ of the total photon flux.

Partial cation and anion yields were obtained with a magnetic mass spectrometer described previously [31]. The instrument consists of a $180^{\circ}$ magnetic mass spectrometer with a resolution of 1 mass in 50, an electrostatic lens to focus the ions created in the interaction region onto the entrance slit of the spectrometer, and an effusive-jet gas cell containing push and extraction plates to move the ions from the interaction region into the lens. Ions are detected with a channel electron multiplier (CEM) at the exit slit of the spectrometer. The polarities of the lens, magnetic field, and CEM may be switched, allowing measurement of either cations or anions produced by photofragmentation. In addition, an analog signal from a capacitance manometer was recorded simultaneously with the ion signal and incident photon flux to monitor target gas pressure for a point-by-point normalization of the recorded spectra. The working pressure in the target chamber was approximately $1 \times 10^{-5}$ Torr, and the chamber was isolated from the beamline vacuum $\left(1 \times 10^{-9}\right.$ Torr $)$ by a differential pumping system $\left(2 \times 10^{-8}\right.$ Torr).

The commercially obtained sample (Apogee Technology) had 98.5\% purity, and was used without further purification.

In order to obtain reasonable statistics, most of the spectra presented here are the 
sum of several recordings, separately normalized, especially the weaker channels such as the doubly charged cations and the anions.

\section{Results and discussion}

The electronic structure of $(\mathrm{CN})_{2}$ generally agreed upon is the following $[7,8,10]$ :

$$
\left(1 \sigma_{g}\right)^{2}\left(1 \sigma_{u}\right)^{2}\left(2 \sigma_{g}\right)^{2}\left(2 \sigma_{u}\right)^{2}\left(3 \sigma_{g}\right)^{2}\left(3 \sigma_{u}\right)^{2}\left(4 \sigma_{g}\right)^{2}\left(1 \pi_{u}\right)^{4}\left(4 \sigma_{u}\right)^{2}\left(5 \sigma_{g}\right)^{2}\left(1 \pi_{g}\right)^{4}\left(2 \pi_{u}\right)^{0}\left(2 \pi_{g}\right)^{0}
$$

where $\left(2 \pi_{u}\right)$ and $\left(2 \pi_{g}\right)$ are the two lowest unoccupied molecular orbitals (LUMO). Due to the presence of two carbon and two nitrogen 1s orbitals, a gerade-ungerade splitting is expected for both pairs. Using restricted Hartree-Fock SCF calculations, Bell [9] obtained a gerade-ungerade splitting of $28 \mathrm{meV}$ for $\mathrm{C} 1 s$. For $\mathrm{N} 1 s$, he reports no splitting. The consequence is that there are no symmetry restrictions to inner shell excitations to virtual molecular orbitals. Furthermore, the number of possible shape resonances is also related to such parity splitting, in the sense that continuum states with both $\mathrm{g}$ and $\mathrm{u}$ symmetry are in principle reachable. Kreile et al. [24], using a LCAO/MSX $\alpha$ method, calculated that there are four shape resonances for valence photoionization, with $\pi_{g}, \sigma_{u}, \sigma_{g}$, and $\sigma_{u}$ symmetry, corresponding to the virtual orbitals obtained in minimal basis set description: $2 \pi_{g}, 5 \sigma_{u}, 6 \sigma_{g}$ and $6 \sigma_{u}$ respectively. Excitation to the unoccupied $2 \pi_{g}$ orbital results in bound state. Lynch et al. [26] also suggest that there are four shape resonances for valence ionization, but that these are a $\sigma_{u}$ resonance due to a $l=3$ resonance for the $\mathrm{C}-\mathrm{C}$ bond, a $\sigma_{u}$ and a $\sigma_{g}$ resonance, which are symmetric and anti-symmetric combinations of the $l=3$ resonance in the $\mathrm{C}-\mathrm{N}$ bonds, and an unusual shape resonance in the $\pi_{u}$ continuum. The presence of a core hole may drag the $\mathrm{C}-\mathrm{C} \sigma_{u}$ resonance below the ionization threshold. Therefore the absorption spectra and consequently the partial ion yields spectra display a wealth of above-threshold features. We will suggest a new interpretation for some of such features at both $\mathrm{C}$ and $\mathrm{N} K$-edges.

\subsection{K-edge}

At the $\mathrm{C} K$ edge 11 different charged fragments were observed:

- eight singly charged cations: $\mathrm{C}_{2} \mathrm{~N}_{2}^{+}, \mathrm{C}_{2} \mathrm{~N}^{+}, \mathrm{CN}_{2}^{+}, \mathrm{CN}^{+}, \mathrm{C}_{2}^{+}, \mathrm{N}_{2}^{+}, \mathrm{C}^{+}, \mathrm{N}^{+}$,

- two doubly-charged cations: $\mathrm{C}^{++}, \mathrm{N}^{++}$,

- and one anion: $\mathrm{CN}^{-}$.

Additionally, there is a fragment with a mass-to-charge ratio of $13 \mathrm{u} / \mathrm{e}$, which could correspond to $\mathrm{CN}^{++}$or ${ }^{13} \mathrm{C}^{+}$. The spectrum of this fragment is very similar to ${ }^{12} \mathrm{C}^{+}$, and its relative intensity is close to the known isotopic ratio of ${ }^{12} \mathrm{C}^{+}$and ${ }^{13} \mathrm{C}^{+}$, why we assign it to the ${ }^{13} \mathrm{C}^{+}$fragment. There are no dramatic differences among the various yields for the positive fragments, therefore we will show only a selected few. However, 
we will show that some trends can be identified which are useful for the spectral interpretation.

In Fig.1, we show the total ion yield (equivalent to absorption) obtained as the sum of all detected ions. The insert in Fig. 1 shows a blow-up of the 289-296 eV region. The previously reported electron energy loss spectrum obtained at the $\mathrm{C} K$ edge with an energy resolution of $0.35 \mathrm{eV}$ [11] was interpreted on the ground of the following assignment: the lowest-energy and most intense feature at photon energy of $286.3 \mathrm{eV}$ was assigned to a transition from the C $1 s$ level(s) to the $2 \pi_{u}$ unoccupied orbital. According to the calculations in [8], the other virtual orbital of $\pi$ symmetry, $2 \pi_{g}$, should be separated from $2 \pi_{u}$ by $5 \mathrm{eV}$, and therefore the related transition was assigned to the peak at $291.7 \mathrm{eV}$. In the intermediate region, another multiple feature is present, which the authors in [11] attribute to a transition to the $3 s$ Rydberg state. Benefiting from a much higher energy resolution, we can suggest a more accurate assignment.

In the spectrum, the $2 \pi_{u}$ feature exhibits a strong vibrational progression, not resolved in the electron energy loss spectrum [11], with a spacing of the peaks of approximately $0.27 \mathrm{eV}$. In the ${ }^{1} \Sigma_{g}^{+}$ground state of cyanogen, the symmetric and antisymmetric $\mathrm{C}-\mathrm{N}$ stretch vibrations have energies of $289 \mathrm{meV}$ and $268 \mathrm{meV}$, respectively [32]. The $2 \pi_{u}$ orbital has anti-bonding $\mathrm{C}-\mathrm{N}$ character $[8,9]$, why a lowering of the $\mathrm{C}-\mathrm{N}$ stretch vibrational frequencies compared to the ground state possibly could be expected. The vibrational splitting can also be compared the experimentally determined energy of the $\mathrm{C}-\mathrm{N}$ stretch in the radical OCCN, $0.26 \mathrm{eV}$ [33], or the calculated energies of the symmetric and anti-symmetric $\mathrm{C}-\mathrm{N}$ stretch vibrations in the $\mathrm{NCCN}^{-}$anion, $247 \mathrm{meV}$ and $225 \mathrm{meV}$ [34], since these molecules and core-excited cyanogen have isoelectronic valence shells. Because the twofold degeneracy of the ${ }^{1} \Pi_{u}$ excited state will be removed by vibronic coupling through the Renner-Teller effect, we can not unequivocally attribute the peaks to either symmetric or anti-symmetric $\mathrm{C}-\mathrm{N}$ stretch vibrations. For instance, in ethyne, another linear four-membered molecule, vibronic coupling effects in the $\mathrm{C} 1 s \rightarrow \pi^{*}$ excitation lead to a domination of bending modes, but also allow both symmetric and anti-symmetric $\mathrm{C}-\mathrm{H}$ stretch vibrations [35]. From attempts to curve fit the spectrum with Voigt profiles, where the Gaussian contribution primarily comes from the uncertainty in the photon energy and the Lorentzian contribution comes from the lifetime of the core hole, it is clear that excitation of a single vibrational mode is not sufficient to describe the $2 \pi_{u}$ feature.

In the photon energy region 290-295 eV, we resolve several peaks which were overlapping in the electron energy loss spectra, and we obtain photon energies of the resonances which are slightly different from [11]. The ionization threshold is set at 294.5 $\mathrm{eV}$, and there is one extra resonant feature at threshold which is tentatively discussed as related to a shape resonance of mainly $\sigma_{C-N}$ character. All energies and peak assignment are reported in Table 1. The intense peak observed at $292.15 \mathrm{eV}$ shows a clear vibrational progression with a spacing of $0.28 \mathrm{eV}$, close to the vibrational spacing 
measured on the $2 \pi_{u}$ resonance, and we assign it to the $2 \pi_{g}$ molecular state. Based on calculated energies of the Rydberg states obtained using the quantum defects $\delta \mathrm{s} \sigma=1.03$ and $\delta \mathrm{p} \sigma=0.67$ given in [8], we can assign the Rydberg states to the observed peaks as follow: $3 s$ at $290.91 \mathrm{eV}, 3 p$ at $291.75 \mathrm{eV}, 4 s$ at $293.02 \mathrm{eV}$, and an additional state not observed in the Electron loss spectrum, namely $4 p$ at $293.37 \mathrm{eV}$. The $4 p$ state in our spectrum shows a structure we assign to a vibrational progression with a spacing of $0.24 \mathrm{eV}$, consistent with the $\mathrm{C}-\mathrm{N}$ stretching mode.

Although our assignment refers to pure Rydberg states denomination, low Rydberg states are commonly described as Rydberg-valence mixed character [36]. In Fig. 2 we show the ion yields measured for $\mathrm{C}_{2} \mathrm{~N}_{2}^{+}, \mathrm{CN}^{+}$, and $\mathrm{N}^{+}$. The general appearance of the partial yield spectra in the energy region below threshold is quite similar, but there are some significant differences which can aid in achieving a detailed spectral assignment. By comparing the partial yields shown in Fig.2, we notice an effect we have reported for several other systems [27, 28], and namely that the relative intensity of the Rydberg series is higher for the smaller fragments. This is due to the fact that the excitations to Rydberg states decay mainly through spectator processes, and therefore the final states are mostly dissociative in nature. In the present case, we can exploit this observation in the following way: we notice an increase of the spectral features in the photon energy region between 292.7 and $293.7 \mathrm{eV}$, but the relative intensity of the broad feature with the first maximum at $290.91 \mathrm{eV}$, which in [11] is attributed to a transition to the Rydberg $3 s$ state, does not mimic that of the sharp peaks at higher photon energy. We can then deduce that the feature is not purely Rydberg in nature, but has an admixture of virtual valence orbitals with $\sigma$ symmetry. This mixing has been reported for acetylene, in which we were able to identify the mixing by the relative intensity changes in the ion yields as a function of fragmentation [27]. We propose the same explanation for the present case. We also notice that this feature has additional structure, with a spacing of about $0.8 \mathrm{eV}$. This is not compatible with a vibrational mode, and we attribute this to that the above mentioned mixing results in more than one level.

In the above-threshold region, the literature spectrum shows two features at 306 and $313 \mathrm{eV}$, assigned as $\sigma_{C-N}$ and $\sigma_{C-C}$ shape resonances, plus the already mentioned broad feature exactly at threshold (located at $294.5 \mathrm{eV}$ by photoemission experiments) [11]. The assignment of the two continuum features far above threshold, centred at approximately 306 and $314 \mathrm{eV}$ in our measurement, as related to shape resonances mainly localized on the $\mathrm{C}-\mathrm{N}$ or the $\mathrm{C}-\mathrm{C}$ bond is reasonable, based upon the simple argument about the length and strength of the bonds. Although we have never agreed with the "bond length with a ruler" model (see for instance [37]), it is quite natural to relate the strength of the photoelectron trapping responsible for the shape resonance phenomenon to the depth of the potential well and therefore to the strength of the chemical bond, in a system with more than one type of bond. The fact that the continuum feature assigned to the $\sigma_{C-C}$ shape resonance is not observed at the $\mathrm{N} K$ edge confirms this 
assignment. The relative intensities of the shape resonances are enhanced in the doubly charged fragments, interestingly for the $\sigma_{C-C}$ particularly in the $\mathrm{N}^{++}$fragment (not shown). We have observed such a tendency also in other molecules [27]. As for the number of shape resonances predicted by the calculations, at least four [24, 26], we can comment that some of these are likely to be pushed below threshold by the presence of the core hole, and therefore we can relate some of them to the above assigned virtual orbitals with either $\pi$ or $\sigma$ character. In our opinion, the open question is the assignment of the spectral feature at threshold.

To obtain a better insight into its nature, we report in Fig. 3 the partial yields for the only negative ion detected at the $\mathrm{C} K$ edge, $\mathrm{CN}^{-}$, and its positive counterpart $\mathrm{CN}^{+}$, in the 289-320 eV energy region. One of the criteria we have established to assign shape resonances is the fact that in small molecules they do not show up in the negative ion yields because it is unlikely to start from a doubly positive charged fragment and produce a negatively charged one [12]. We can see that while the continuum resonances above threshold do not appear in the $\mathrm{CN}^{-}$yield, but they do in the $\mathrm{CN}^{+}$yield, the feature at threshold shows up clearly in both yields and appears to be enhanced in the $\mathrm{CN}^{-}$spectrum. We therefore conclude that it is not a likely to be a shape resonance, but rather a neutral doubly excited state. There is a satellite threshold opening at about $303 \mathrm{eV}$ [11], and the feature at threshold is likely to correspond to a double excitation leading to that threshold. The relative distance $(8.5 \mathrm{eV})$ is similar to the distance between the $2 \pi_{u}$ peak at $286.3 \mathrm{eV}$ and the mainline threshold $(8.2 \mathrm{eV})$, which supports such an assignment. We will show that this argument works even more clearly at the $\mathrm{N} K$ edge (see discussion below).

\subsection{Formation of $\mathrm{N}_{2}^{+}$}

Among the different ions produced after core excitation of $(\mathrm{CN})_{2}$, the singly charged $\mathrm{N}_{2}^{+}$ fragment is observed. Formation of $\mathrm{N}_{2}^{+}$requires a strong rearrangement of the molecular structure as it involves the breaking of two triple bonds between the nitrogen and the carbon atoms and the formation of a new bond between the two nitrogen atoms. Other examples of intramolecular atomic rearrangement were previously observed, involving the breaking of a $\mathrm{H}-\mathrm{X}$ bond and formation of another bond during the reconfiguration of the electronic structure of the molecule following photoionization: formation of $\mathrm{H}_{2}^{+}$ from $\mathrm{H}_{2} \mathrm{O}$ [38]; and $\mathrm{H}_{2}^{+}, \mathrm{H}_{3}^{+}$and $\mathrm{HCl}^{+}$from $\mathrm{CH}_{3} \mathrm{Cl}$ [28]. In both of these examples, rearrangement is possible due to the high mobility of protons and is aided by vibrational excitation. More recently, we reported the observation of the formation of $\mathrm{O}_{2}^{+}$after core excitation in formic acid [20]. Such rearrangement is surprising because it requires the breaking of two bonds, the two oxygen atoms being attached to the same carbon in the molecule, and the formation of a bond between the two oxygen atoms. In the case of cyanogen the formation of $\mathrm{N}_{2}^{+}$appears even more surprising because the two nitrogen atoms do not share the same carbon atom but are spatially separated by the $\mathrm{C}-\mathrm{C}$ bond. 
In Fig. 4, we compare the partial ion yield for $\mathrm{N}^{+}$and $\mathrm{N}_{2}^{+}$in the energy region of the $2 \pi_{u}$ resonance, 285-289 eV, and in the Rydberg region, 289-296 eV. The two spectra were arbitrarily normalized to the intensity of the $2 \pi_{u}$ resonance to permit relative intensity comparison. First of all, the differences between the spectra speak against the origin of $\mathrm{N}_{2}^{+}$being a recombination fragment, in which case the $\mathrm{N}_{2}^{+}$yield would be expected to mimic that of $\mathrm{N}^{+}$. We observe in the $\mathrm{N}_{2}^{+}$partial ion yield an enhancement of the high vibrational levels along the $2 \pi_{u}$ resonance (top panel in Fig. 4, in particular the $v=4$ and $v=5 \mathrm{C}-\mathrm{N}$ stretch vibrational states which are found very weak in the $\mathrm{N}^{+}$partial yield. After excitation to higher vibrational states in the core-excited neutral state, the electron decay reaches final states in turn highly vibrationally excited, where the vibrational excitation helps the wavepacket to pass the potential barrier to formation of $\mathrm{N}_{2}^{+}$. This could suggest an assignment of the vibrational progression as mainly due to the anti-symmetric $\mathrm{C}-\mathrm{N}$ stretch mode, since this would lower the symmetry of the molecule, and the symmetric stretch would also distance the nitrogen atoms from each other. In addition, excitation of bending modes can be expected, only their vibrational frequencies are lower, and would not be resolved in the spectrum. This observation confirms the crucial role of vibrational excitation in the formation of such ionic species after core excitation.

In the bottom panel of fig. 4 , we observe in the $\mathrm{N}_{2}^{+}$partial yield an enhancement of the peak at $290.91 \mathrm{eV}$ that we assigned to the transition to the $3 s$ Ryberg state. We note that the transition to the $2 \pi_{g}$ molecular orbital is at the same relative intensity compared to the $\mathrm{N}^{+}$partial yield. Therefore, we interpret this enhancement as a confirmation of the Ryberg-valence mixed character of the $3 s$ state. Similar observations were made at the $\mathrm{N} K$ edge, although due to the presence of a $\mathrm{N}_{2}$ contamination in the sample, it was not possible to observe the high vibrational states along the $2 \pi_{u}$ resonance as they overlap with the very intense $\mathrm{N}_{2} \mathrm{~N} 1 s \rightarrow 2 \pi$ resonance which appears at 400.87 $\mathrm{eV}$ in the $\mathrm{N}_{2}^{+}$partial yield. We also note that for the other fragment which requires a rearrangement of the the molecular structure, $\mathrm{CN}_{2}^{+}$, the relative intensity of the $3 \mathrm{~s}$ feature is rather decreased compared to the $2 \pi_{g}$ feature, both at the C $K$ and N $K$ edges, and no enhancement of higher vibrational excitations can be observed at the $2 \pi_{u}$ resonance.

\section{3. $N$ K-edge}

At the N $K$ edge, 13 different charged fragments were detected:

- eight singly charged cations: $\mathrm{C}_{2} \mathrm{~N}_{2}^{+}, \mathrm{C}_{2} \mathrm{~N}^{+}, \mathrm{CN}_{2}^{+}, \mathrm{CN}^{+}, \mathrm{C}_{2}^{+}, \mathrm{N}_{2}^{+}, \mathrm{C}^{+}, \mathrm{N}^{+}$,

- three doubly charged cations: $\mathrm{C}_{2} \mathrm{~N}^{++}, \mathrm{C}^{++}, \mathrm{N}^{++}$,

- and two anions: $\mathrm{CN}^{-}$and $\mathrm{C}^{-}$.

Again, we also observe a fragment with mass-to-charge ratio of $13 \mathrm{u} / \mathrm{e}$, which we assign to the ${ }^{13} \mathrm{C}^{+}$fragment. As for the $\mathrm{C} K$ edge, we show in Fig. 5 the total ion yield spectrum obtained as the sum of all detected fragments. The literature assignment for 
this edge is analogous to the one already reported for the $\mathrm{C} K$ edge. In particular, the features in the electron energy loss spectrum are assigned as follows [11]: the lowestenergy and most intense feature at a photon energy of $398.9 \mathrm{eV}$ is assigned to a transition from the $\mathrm{N} 1 s$ level(s) to the $2 \pi_{u}$ virtual molecular orbital, a peak at $403.4 \mathrm{eV}$ is related to a transition to the $3 s$ Rydberg state, a peak at $404.7 \mathrm{eV}$ is assigned as overlapping transitions to the $2 \pi_{g}$ virtual orbital and the Rydberg $3 p$ state, and a peak at $405.6 \mathrm{eV}$ is assigned to a transition to the $4 s$ Rydberg state. The ionization threshold is located at $407.4 \mathrm{eV}$ as measured by photoelectron spectroscopy. An intense resonant feature at threshold is assigned as a $\sigma_{C-N}$ shape resonance, and another feature in the ionization continuum at $419.3 \mathrm{eV}$ is assigned to another $\sigma_{C-N}$ shape resonance. On the ground of arguments similar to those we already reported for the $\mathrm{C} K$ edge, we can propose a more complete assignment for the below-threshold features, based on our better experimental conditions. The observed energies and suggested assignments are reported in Table 1.

Just as in the $\mathrm{C} K$ edge case, the $2 \pi_{u}$ feature exhibits a vibrational progression with a splitting between the peaks of approximately $0.27 \mathrm{eV}$, which we assign as coming from $\mathrm{C}-\mathrm{N}$ stretch vibrations. Also here, the width and line shape of the individual peaks are not compatible with a single vibrational progression being excited.

We can identify and assign the following features: the broad multi-structured feature with a maximum at 403.45 as transition(s) to the $3 s$ Rydberg state mixed with virtual orbitals of the same symmetry; the peak at $404.5 \mathrm{eV}$ as a transition to the $3 p$ state, and that at $404.95 \mathrm{eV}$ as a transition to the $2 \pi_{g}$ virtual molecular orbital; the peak at 405.60 is a transition to the $4 s$ state; and finally the transition at $405.95 \mathrm{eV}$ is the $4 p$ state, with possible vibrational levels. We use the same argument reported above for the $\mathrm{C} K$ edge for the assignment of the $3 s$ Rydberg level as mixed to states with molecular orbital character. We show in Fig. 6 the partial ion yields for $\mathrm{C}_{2} \mathrm{~N}_{2}^{+}, \mathrm{CN}^{+}$, and $\mathrm{N}^{+}$. We observe a trend in relative intensity as a function of fragmentation, similar to that at the $\mathrm{C} K$-edge, but more pronounced. The states with pure Rydberg character (namely $4 s$ and $4 p$ ) are more prominent for the smaller fragments, and therefore the states in the spectral region of the Rydberg $3 s$, which do not mimic this behavior, are not solely or mainly Rydberg in character.

In Fig. 7, we report the yields for the two negative ions detectable around the $\mathrm{N}$ $K$-edge, namely $\mathrm{CN}^{-}$and $\mathrm{C}^{-}$, together with their positive counterparts. In analogy with the $\mathrm{C} K$ edge, we notice that the feature at threshold is clearly visible in the negative ion yields. However, due to poor statistics it is less clear in these yields if the continuum features discussed above as attributable to shape resonances are present or not. We may argue again that while the feature at $420 \mathrm{eV}$ is likely to be a $\sigma_{C-N}$ shape resonance, the threshold feature is related to a doubly excited state leading to a satellite threshold rather than to a shape resonance. A satellite with ionization threshold around $416 \mathrm{eV}$ has been identified [11], which supports this interpretation. 


\section{Conclusion}

We have recorded partial-ion-yield spectra of the $(\mathrm{CN})_{2}$ molecule in the vicinity of the $\mathrm{C} 1 s$ and $\mathrm{N} 1 s$ ionization thresholds. From characteristics in the cationic partial yield spectra in combination with improved resolution, a more detailed assignment compared to the only published core-excitation spectrum has been possible. The $\mathrm{N}_{2}^{+}$ fragment, which requires substantial molecular reorganization, has been observed, and its formation was shown to be aided by vibrational excitation. Assignment of features due to doubly excited states or shape resonances in the ionization continuum has been possible by the suppression of the shape-resonant contribution in anion-yield spectra. We have observed similar behaviour in several other small molecules, showing that negative-ion-yield measurements are generally useful in X-ray spectroscopy of molecules.

\section{Acknowledgement}

The authors thank the staff of the ALS for their excellent support. Support from the National Science Foundation under NSF Grant No. PHY-05-55699 is gratefully acknowledged. This work was performed at the Advanced Light Source, which is supported by DOE (DE-AC03-76SF00098).

[1] Wagner R M and Schleicher D G 1997 Science 1918

[2] Kleine M, Wyckoff S, Wehinger P A, and Peterson B A 1994 Astrophys. J. 436885

[3] Baker C and Turner D W Proc. 1968 R. Sot. London Ser. A 30819

[4] Turner D W, Baker C, Baker A D, and Brundle C R 1970 Molecular Photoelectron Spectroscopy pp. 345-355 (London: Wiley-Interscience)

[5] Åsbrink L, von Niessen W, and Bieri G 1980 J. Electron Spectrosc. Relat. Phenom. 2193

[6] Hochlaf M, Baer T, Qian X-M, and Ng C Y 2005 J. Chem. Phys. 123144302

[7] Cederbaum L S, Domcke W, and von Niessen W 1975 Chem. Phys. 10459

[8] Fridh C, Åsbrink L, and Lindholm E 1978 Chem. Phys. 27169

[9] Bell S 1979 Chem. Phys. Lett. 67498

[10] Cederbaum L S, Domcke W, Schirmer J, and Köppel H 1980 J. Chem. Phys. 721348

[11] Hitchcock A S and Brion C E 1979 Chem. Phys. 37 319; and references therein.

[12] Stolte W C, Hansen D L, Piancastelli M N, Dominguez Lopez I, Rizvi A, Hemmers O, Wang H, Schlachter A S, Lubell M S, and Lindle D W 2001 Phys. Rev. Lett. 864504

[13] Hansen D L, Stolte W C, Hemmers O, Guillemin R, and Lindle D W 2002 J. Phys. B: At. Mol. Opt. Phys. 35 L381

[14] Öhrwall G, Sant'Anna M M, Stolte W C, Dominguez-Lopez I, Dang L T N, Schlachter A S, and Lindle D W 2002 J. Phys. B: At. Mol. Opt. Phys. 354543

[15] Stolte W C, Sant'Anna M M, Öhrwall G, Dominguez-Lopez I, Piancastelli M N, and Lindle D W 2003 Phys. Rev. A 68022701

[16] Dang L T N, Stolte W C, Öhrwall G, Sant'Anna M M, Dominguez-Lopez I, Schlachter A S, and Lindle D W 2003 Chem. Phys. 28945

[17] Yu S -W, Stolte W C, Öhrwall G, Guillemin R, Piancastelli M N, and Lindle D W 2003 J. Phys. B: At. Mol. Opt. Phys. 361255

[18] Yu S -W, Stolte W C, Guillemin R, Öhrwall G, Tran I C, Piancastelli M N, Feng R, and Lindle D W 2004 J. Phys. B: At. Mol. Opt. Phys. 373583

[19] Guillemin R, Stolte W C, Dang L T N, Yu S -W, and Lindle D W 2005 J. Chem. Phys. 122094318

[20] Guillemin R, Stolte W C, and Lindle D W 2009 J. Phys. B: At. Mol. Opt. Phys. 42125101

[21] Dehmer J L and Dill D 1975 Phys. Rev. Lett. 35213 
[22] Scully S W J, Mackie R A, Browning R, Dunn K F, and Latimer C J 2002 J. Phys. B: At. Mol. Opt. Phys. 352703

[23] Piancastelli M N, Stolte W C, Guillemin R, Wolska A, Yu S -W, Sant'Anna M M, and Lindle D W 2005 J. Chem. Phys. 122094312

[24] Kreile J, Schweig A, and Thiel W 1983 Chem. Phys. Lett. 100351

[25] Holland D M P, Parr A C, Ederer D L, West J B, and Dehmer J L 1983 Int. J. Mass Spectrom. Ion Phys. 52195

[26] Lynch D L, Dixit S N, and McKoy V 1986 J. Chem. Phys. 845504

[27] Piancastelli M N, Stolte W C, Öhrwall G, Yu S-W , Bull D, Lantz K, Schlachter A S, and Lindle D W 2002 J. Chem. Phys. 117 8264; and references therein.

[28] Céolin D, Piancastelli M N, Guillemin R, Stolte W C, Yu S -W, Hemmers O, and Lindle D W 2007 J. Chem. Phys. 126084309

[29] Tronc M, King C G, and Read F R 1979 J. Phys. B: At. Mol. Phys. 12137

[30] Chen C T, Ma Y and Sette F 1989 Phys. Rev. A 406737

[31] Stolte W C, Guillemin R, Yu S-W, and Lindle D W 2008 J. Phys. B: At. Mol. Opt. Phys. 41 145102

[32] Herzberg G 1991 Molecular Spectra and Molecular Structure, vol. III - Electronic Spectra and Electronic Structure of Polyatomic Molecules (Malabar, FL: Krieger Publishing Company)

[33] McNavage W, Dailey W, and Dai H-L 2004 Can. J. Chem. 82925

[34] Nsangou M, Senent M L, and Hochlaf M 2008 Chem. Phys. 355164

[35] Kempgens B, Itchkawitz B S, Feldhaus J, Bradshaw A M, Köppel H, Döscher M, Gadea F X, Cederbaum L S 1997 Chem. Phys. Lett. 277436

[36] Kosugi N 2005 Brazilian J. Phys. 35957

[37] Piancastelli M N 1999 J. Electron Spectrosc. Relat. Phenom. 100167

[38] Piancastelli M N, Hempelmann A, Heiser F, Gessner O, Rüdel A, and Becker U 1999 Phys. Rev. A 59300 
Table 1. Observed energies and assignment for excitation of $\mathrm{C} 1 s$ and $\mathrm{N} 1 s$ electrons in $(\mathrm{CN})_{2}$ to the $2 \pi$ unoccupied molecular orbitals and Rydberg states. The calculated energies were obtained using the quantum defects $\delta \mathrm{s} \sigma=1.03$ and $\delta \mathrm{p} \sigma=0.67$ [8]. A comparison with Hitchcock and Brion [11] is also included.

\begin{tabular}{lccccccc}
\hline & \multicolumn{3}{c}{ C $K$-edge } & \multicolumn{5}{c}{ N $K$-edge } \\
\hline State & \multicolumn{3}{c}{ Energy (eV) } & \multicolumn{5}{c}{ Energy (eV) } \\
& This & Term & Calc. & Ref. & This & Term & Ref. \\
& study & value & energy & {$[11]$} & study & value & {$[11]$} \\
\hline$(1 s)^{-1} 2 \pi_{u}$ & 286.3 & -8.2 & & 286.3 & 398.9 & -8.5 & 398.9 \\
$(1 s)^{-1} 3 s$ & 290.91 & -3.59 & -3.51 & 290.5 & 403.45 & -3.95 & 403.4 \\
$(1 s)^{-1} 3 p$ & 291.75 & -2.75 & -2.51 & 291.7 & 404.50 & -2.90 & 404.7 \\
$(1 s)^{-1} 2 \pi_{g}(\nu=0)$ & 292.15 & -2.35 & & 291.7 & 404.95 & -2.45 & 404.7 \\
$2 \pi_{g}(\nu=1)$ & 292.43 & & & & & & \\
$2 \pi_{g}(\nu=2)$ & 292.71 & & & & & & \\
$(1 s)^{-1} 4 s$ & 293.02 & -1.48 & -1.54 & 293.1 & 405.60 & -1.80 & 405.6 \\
$(1 s)^{-1} 4 p(\nu=0)$ & 293.37 & -1.13 & -1.23 & & 405.95 & -1.45 & \\
$4 p(\nu=1)$ & 293.61 & & & & & & \\
$(1 s)^{-1} \mathrm{IP}$ & 294.5 & 0 & 0 & 294.5 & 407.4 & 0 & 407.4 \\
\hline
\end{tabular}

\section{FIGURE CAPTIONS}

- Fig. 1: Total ion yield (sum of all fragments detected) measured after photoionization around the $\mathrm{C} K$ edge in $(\mathrm{CN})_{2}$, with suggested assignments. The designation D.E.S. indicates Doubly Excited State.

- Fig. 2: Partial ion yields measured for for $\mathrm{C}_{2} \mathrm{~N}_{2}^{+}, \mathrm{CN}^{+}$, and $\mathrm{N}^{+}$around the $\mathrm{C} K$ edge in $(\mathrm{CN})_{2}$.

- Fig. 3: (Color online) Comparison of the partial ion yields measured for $\mathrm{CN}^{+}$and $\mathrm{CN}^{-}$in the $289-320 \mathrm{eV}$ energy region around the $\mathrm{C} K$ edge in $(\mathrm{CN})_{2}$. The spectra were arbitrarily normalized on the $2 \pi_{g}$ resonance.

- Fig. 4: Comparison of the partial ion yields measured for $\mathrm{N}^{+}$and $\mathrm{N}_{2}^{+}$in the region of the $2 \pi_{u}$ resonance (top panel) and in the Rydberg energy region, 289-296 eV. The spectra were arbitrarily normalized to the intensity of the $2 \pi_{u}$ resonance.

- Fig. 5: Total ion yield (sum of all fragments detected) measured after photoionization around the $\mathrm{N} K$ edge in $(\mathrm{CN})_{2}$, with suggested assignments.

- Fig. 6: Partial ion yields measured for for $\mathrm{C}_{2} \mathrm{~N}_{2}^{+}, \mathrm{CN}^{+}$, and $\mathrm{N}^{+}$around the $\mathrm{N} K$ 

edge in $(\mathrm{CN})_{2}$.

- Fig. 7: (Color online) Comparison of the partial ion yields measured for $\mathrm{CN}^{+}$and $\mathrm{CN}^{-}$, and $\mathrm{C}^{+}$and $\mathrm{C}^{-}$, in the 402-430 eV energy region around the $\mathrm{N} K$ edge in $(\mathrm{CN})_{2}$. The spectra were arbitrarily normalized on the $2 \pi_{g}$ resonance. 


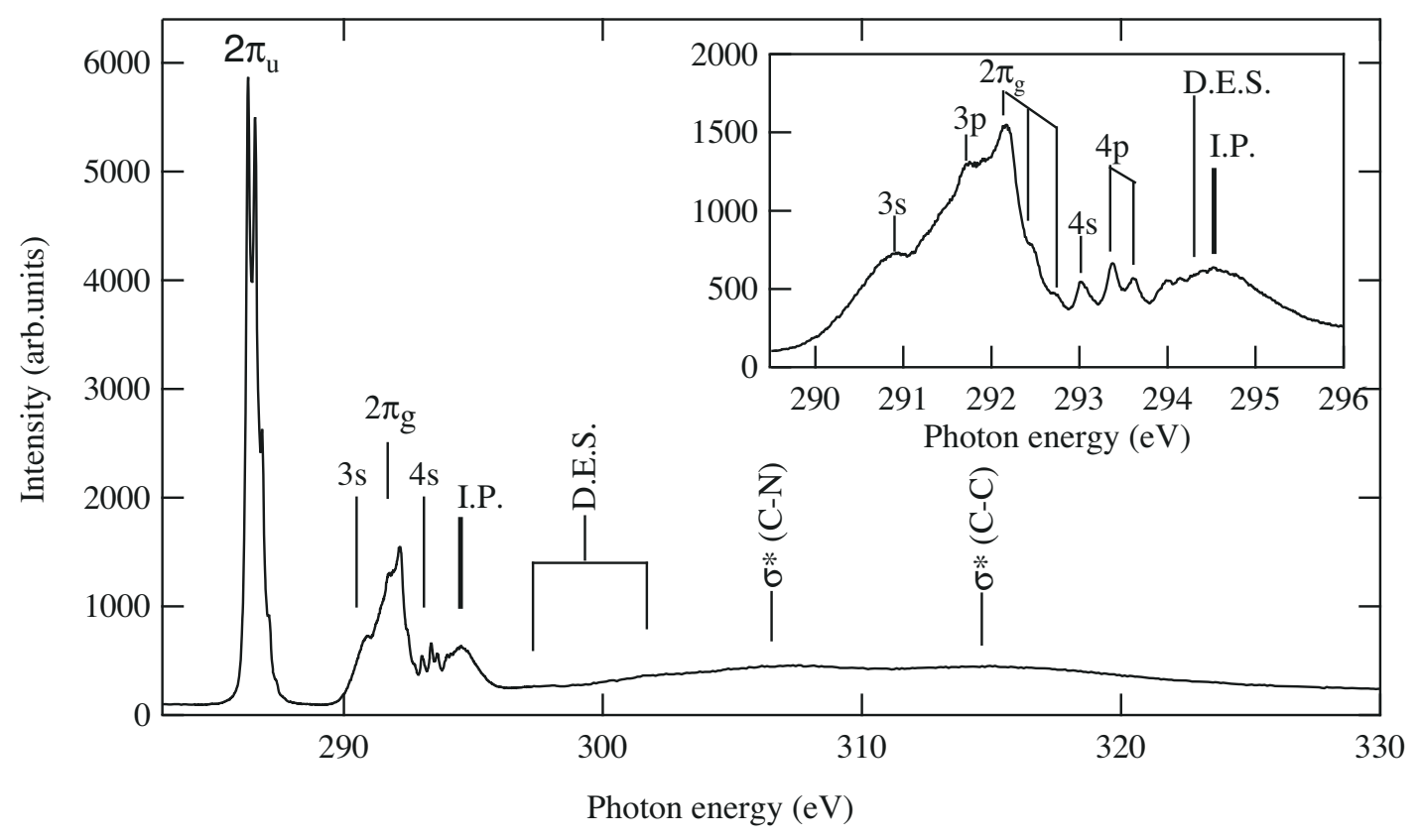

Figure 1. 


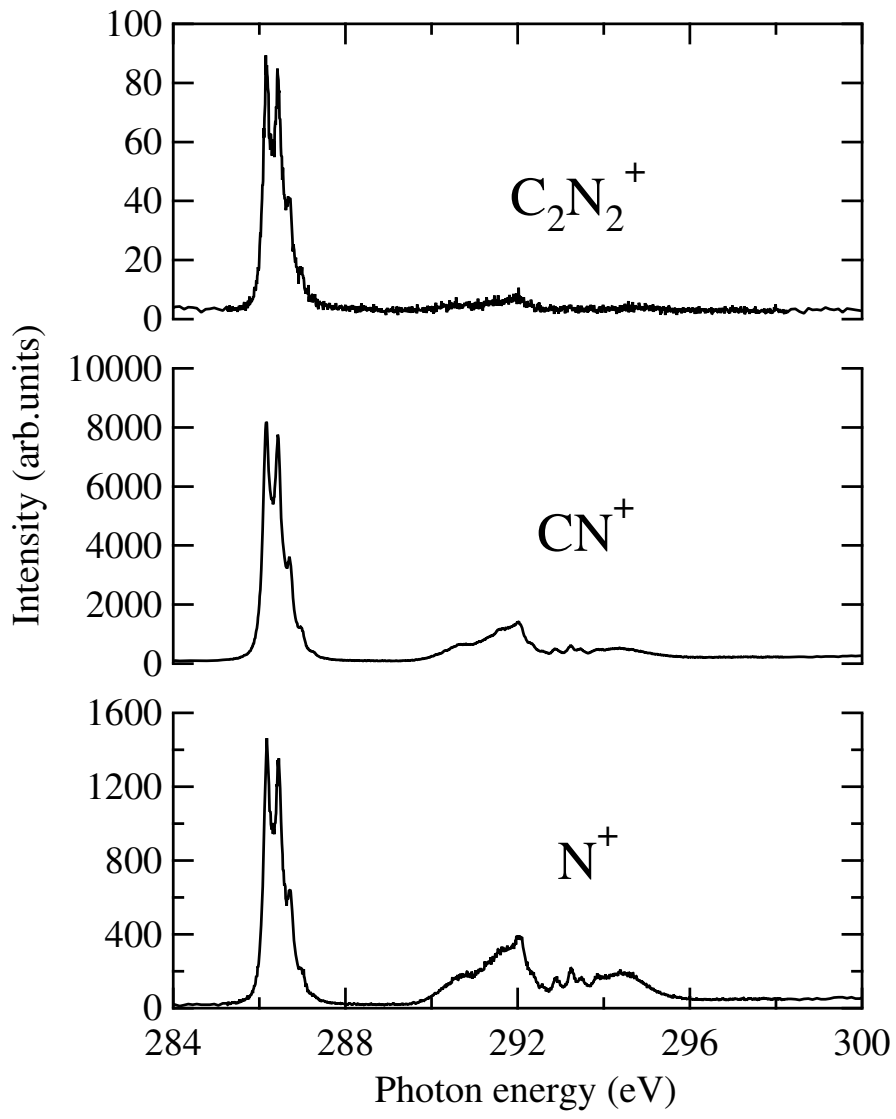

Figure 2. 


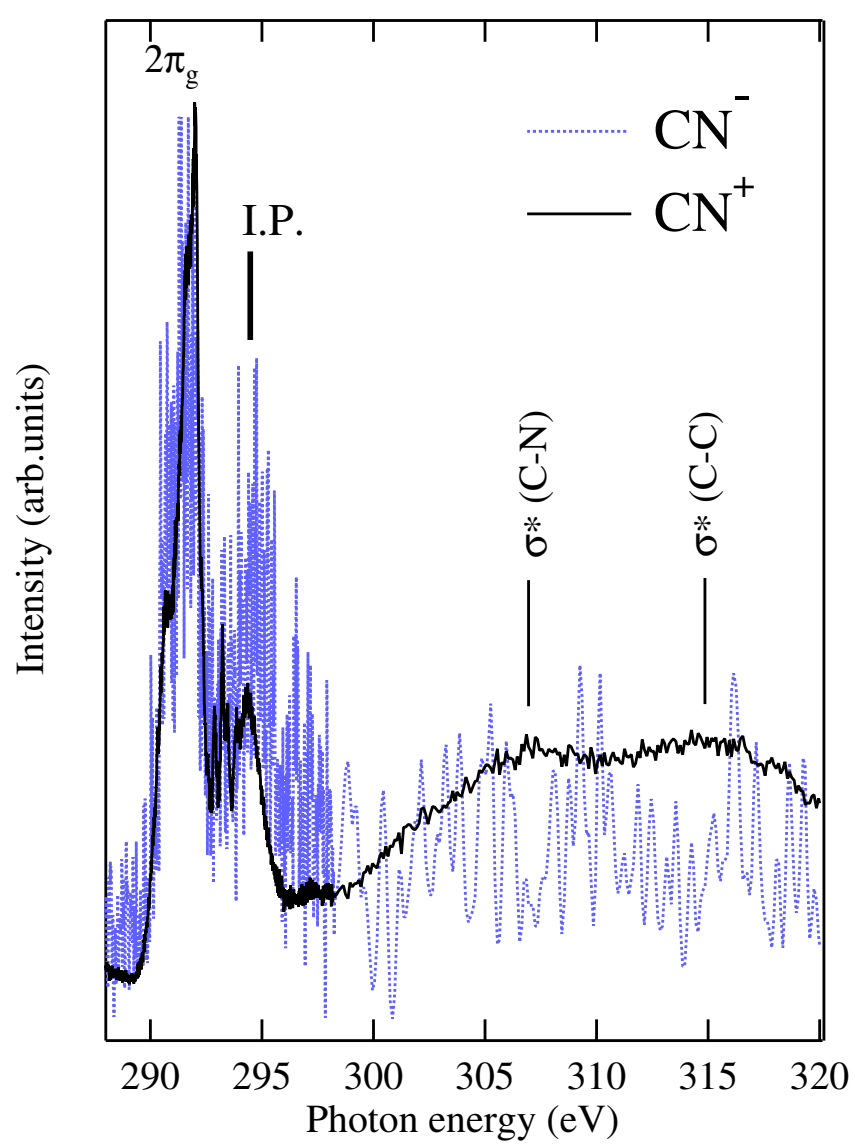

Figure 3. 

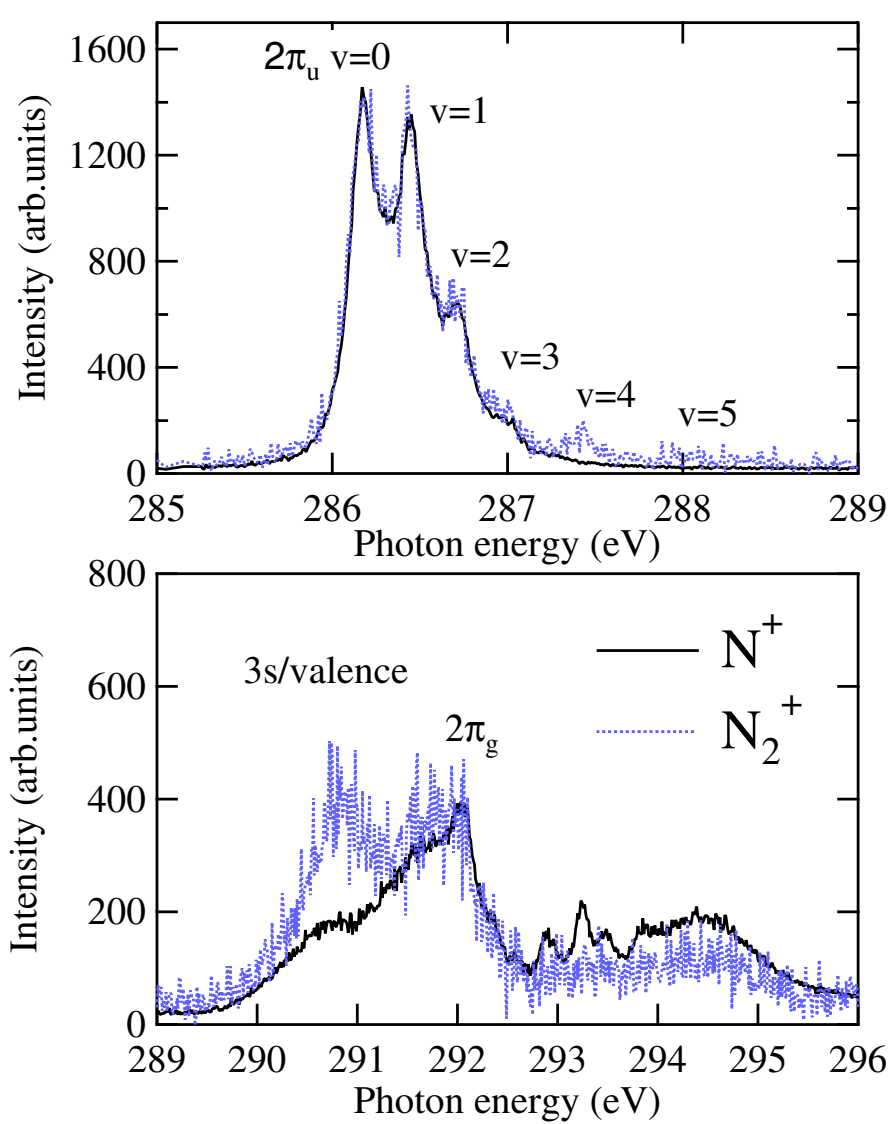

Figure 4. 


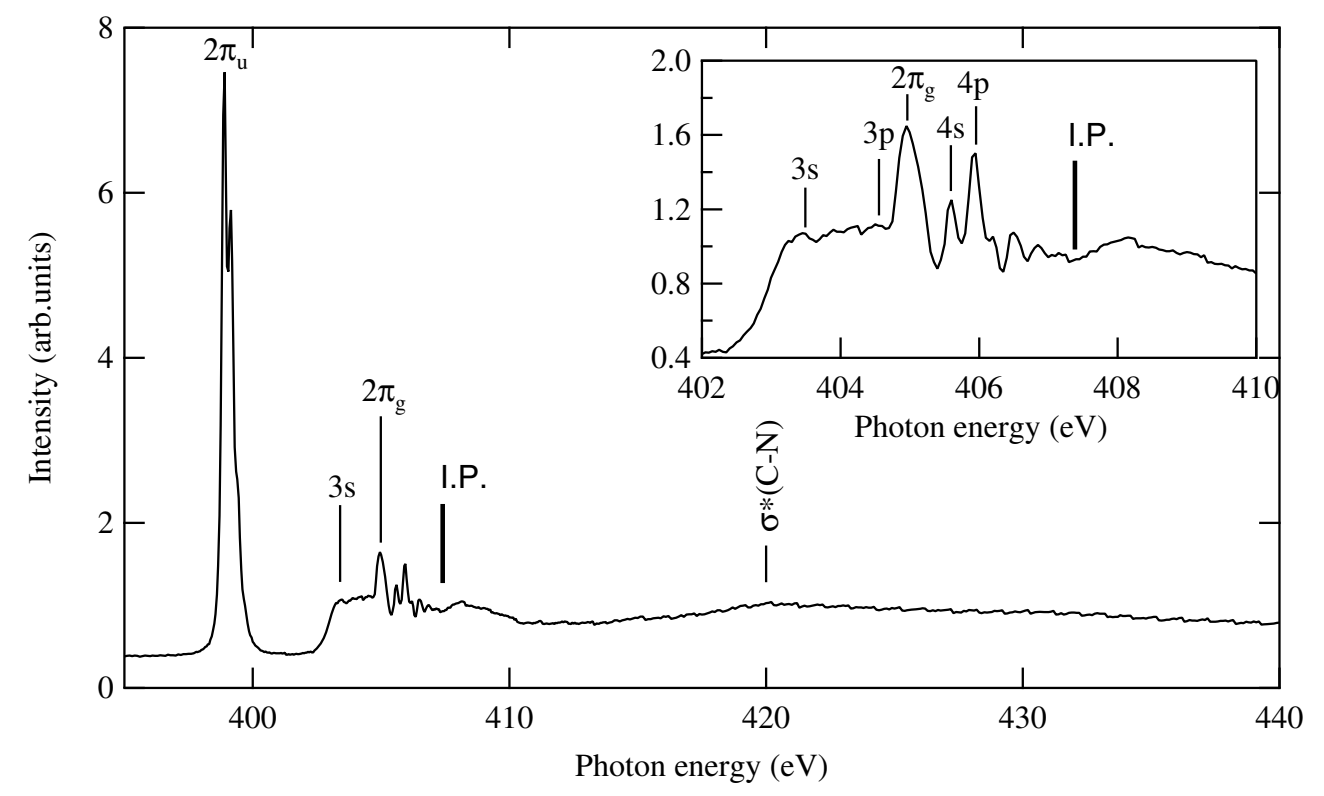

Figure 5. 


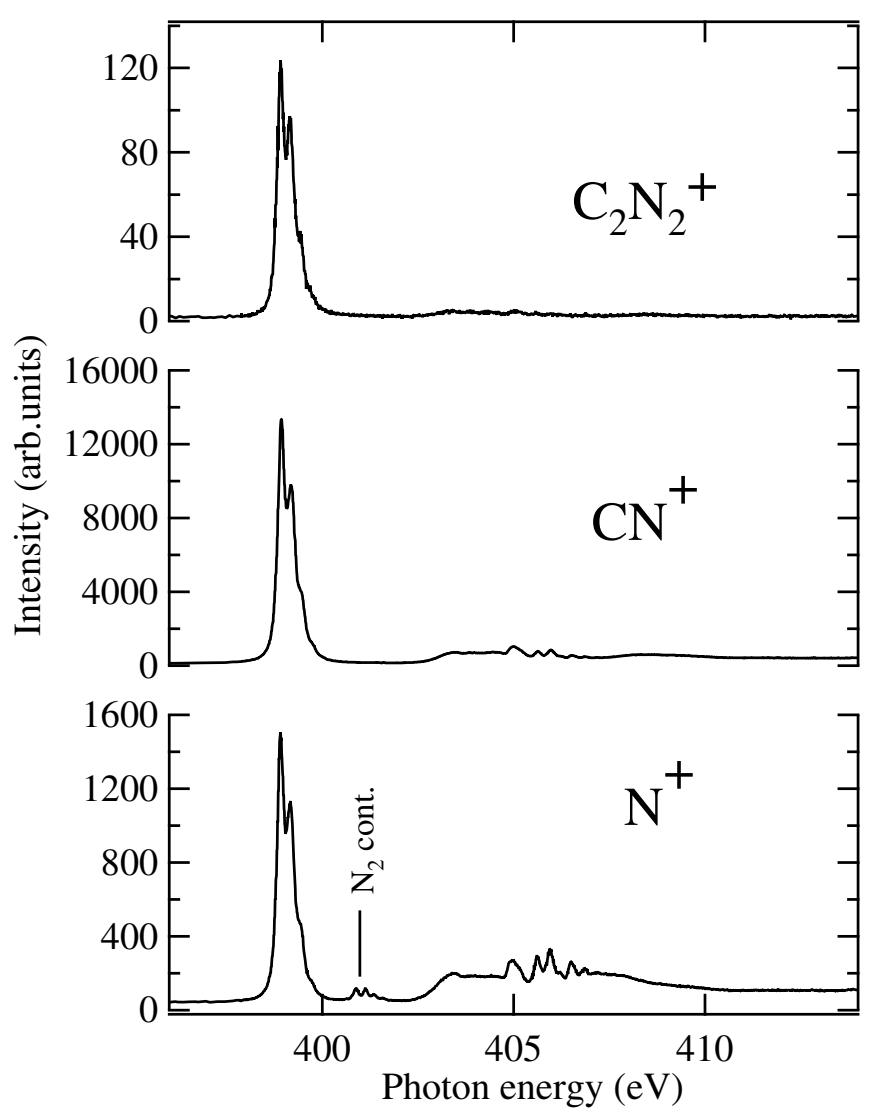

Figure 6. 


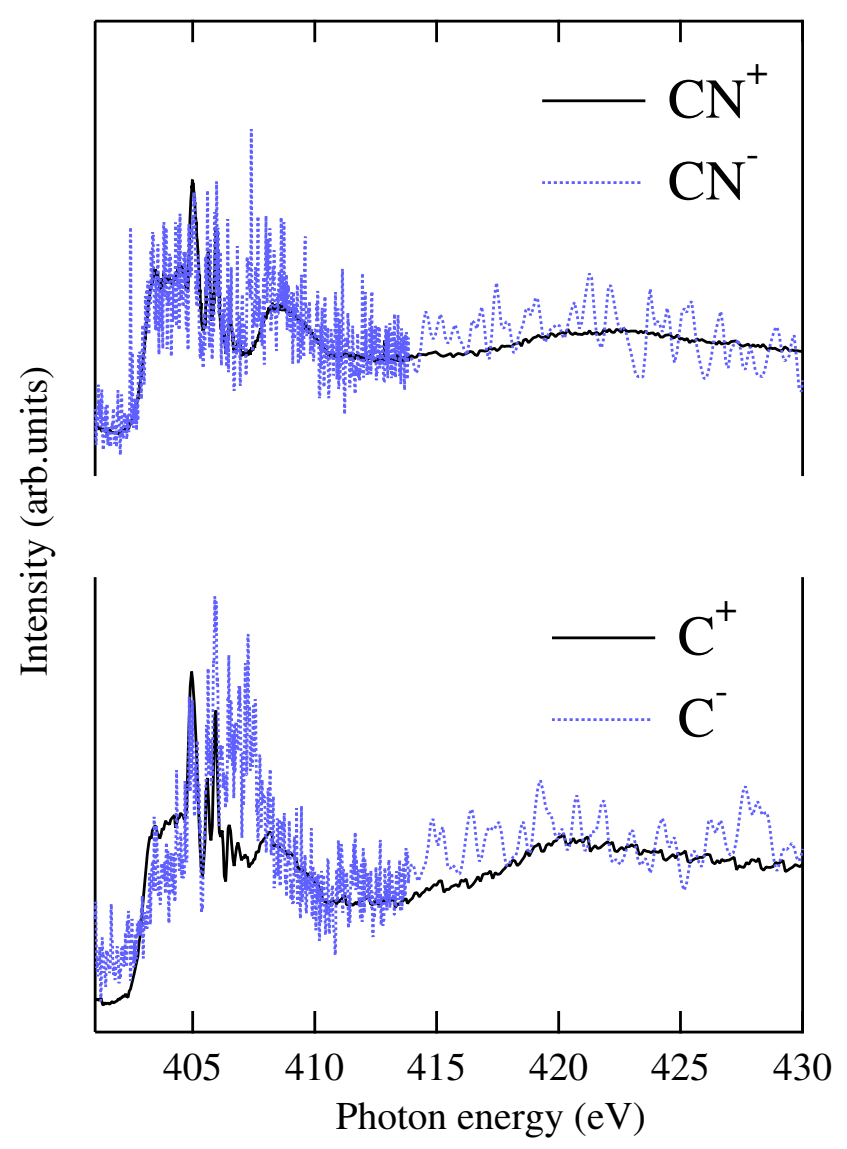

Figure 7. 